\title{
Multiple Positive Solutions for Second-Order p-Laplacian Dynamic Equations with Integral Boundary Conditions
}

\author{
Yongkun Li and Tianwei Zhang \\ Department of Mathematics, Yunnan University, Kunming, Yunnan 650091, China \\ Correspondence should be addressed to Yongkun Li, yklie@ynu.edu.cn
}

Received 13 July 2010; Revised 21 November 2010; Accepted 25 November 2010

Academic Editor: Gennaro Infante

Copyright (C) 2011 Y. Li and T. Zhang. This is an open access article distributed under the Creative Commons Attribution License, which permits unrestricted use, distribution, and reproduction in any medium, provided the original work is properly cited.

We are concerned with the following second-order $p$-Laplacian dynamic equations on time scales $\left(\varphi_{p}\left(x^{\Delta}(t)\right)\right)^{\nabla}+\lambda f\left(t, x(t), x^{\Delta}(t)\right)=0, t \in(0, T)_{\mathbb{T}}$, with integral boundary conditions $x^{\Delta}(0)=0$, $\alpha x(T)-\beta x(0)=\int_{0}^{T} g(s) x(s) \nabla s$. By using Legget-Williams fixed point theorem, some criteria for the existence of at least three positive solutions are established. An example is presented to illustrate the main result.

\section{Introduction}

Boundary value problems with $p$-Laplacian have received a lot of attention in recent years. They often occur in the study of the $n$-dimensional $p$-Laplacian equation, non-Newtonian fluid theory, and the turbulent flow of gas in porous medium [1-7]. Many works have been carried out to discuss the existence of solutions or positive solutions and multiple solutions for the local or nonlocal boundary value problems.

On the other hand, the study of dynamic equations on time scales goes back to its founder Stefan Hilger [8] and is a new area of still fairly theoretical exploration in mathematics. Motivating the subject is the notion that dynamic equations on time scales can build bridges between continuous and discrete equations. Further, the study of time scales has led to several important applications, for example, in the study of insect population models, neural networks, heat transfer, and epidemic models, we refer to [8-10]. In addition, the study of BVPs on time scales has received a lot of attention in the literature, with the pioneering existence results to be found in [11-16].

However, existence results are not available for dynamic equations on time scales with integral boundary conditions. Motivated by above, we aim at studying the second-order 
$p$-Laplacian dynamic equations on time scales in the form of

$$
\left(\varphi_{p}\left(x^{\Delta}(t)\right)\right)^{\nabla}+\lambda f\left(t, x(t), x^{\Delta}(t)\right)=0, \quad t \in(0, T)_{\mathbb{T}}
$$

with integral boundary condition

$$
x^{\Delta}(0)=0, \quad \alpha x(T)-\beta x(0)=\int_{0}^{T} g(s) x(s) \nabla s,
$$

where $\lambda$ is positive parameter, $\varphi_{p}(s)=|s|^{p-2} s$ for $p>1$ with $\varphi_{p}^{-1}=\varphi_{q}$ and $1 / p+1 / q=1$, $\Delta$ is the delta derivative, $\nabla$ is the nabla derivative, $\mathbb{T}$ is a time scale which is a nonempty closed subset of $\mathbb{R}$ with the topology and ordering inherited from $\mathbb{R}, 0$ and $T$ are points in $\mathbb{T}$, an interval $[0, T]_{\mathbb{T}}:=[0, T] \cap \mathbb{T}, f \in C\left([0, T]_{\mathbb{T}} \times \mathbb{R}^{2},[0, \infty)\right)$ with $f(t, 0,0) \neq 0$ for all $t \in[0, T]_{\mathbb{T}}$, $g \in C_{\mathrm{ld}}\left([0, T]_{\mathbb{T}},[0, \infty)\right), \alpha, \beta>0$ with $\alpha-g_{0}>\beta$, and where $g_{0}=\int_{0}^{T} g(s) \nabla s$.

The main purpose of this paper is to establish some sufficient conditions for the existence of at least three positive solutions for BVPs (1.1)-(1.2) by using Legget-Williams fixed point theorem. This paper is organized as follows. In Section 2, some useful lemmas are established. In Section 3, by using Legget-Williams fixed point theorem, we establish sufficient conditions for the existence of at least three positive solutions for BVPs (1.1)-(1.2). An illustrative example is given in Section 4.

\section{Preliminaries}

In this section, we will first recall some basic definitions and lemmas which are used in what follows.

Definition 2.1 (see [8]). A time scale $\mathbb{T}$ is an arbitrary nonempty closed subset of the real set $\mathbb{R}$ with the topology and ordering inherited from $\mathbb{R}$. The forward and backward jump operators $\sigma, \rho: \mathbb{T} \rightarrow \mathbb{T}$ and the graininess $\mu, v: \mathbb{T} \rightarrow \mathbb{R}^{+}$are defined, respectively, by

$$
\sigma(t):=\inf \{s \in \mathbb{T}: s>t\}, \quad \rho(t):=\sup \{s \in \mathbb{T}: s<t\}, \quad \mu(t):=\sigma(t)-t, \quad v(t):=t-\rho(t) .
$$

The point $t \in \mathbb{T}$ is called left-dense, left-scattered, right-dense, or right-scattered if $\rho(t)=t$, $\rho(t)<t$, and $\sigma(t)=t$ or $\sigma(t)>t$, respectively. Points that are right-dense and left-dense at the same time are called dense. If $\mathbb{T}$ has a left-scattered maximum $m_{1}$, defined $\mathbb{T}^{\kappa}=\mathbb{T}-$ $\left\{m_{1}\right\}$; otherwise, set $\mathbb{T}^{\kappa}=\mathbb{T}$. If $\mathbb{T}$ has a right-scattered minimum $m_{2}$, defined $\mathbb{T}_{\kappa}=\mathbb{T}-\left\{m_{2}\right\}$; otherwise, set $\mathbb{T}_{\kappa}=\mathbb{T}$.

Definition 2.2 (see [8]). For $f: \mathbb{T} \rightarrow \mathbb{R}$ and $t \in \mathbb{T}^{\kappa}$, then the delta derivative of $f$ at the point $t$ is defined to be the number $f^{\Delta}(t)$ (provided it exists) with the property that for each $\epsilon>0$, there is a neighborhood $U$ of $t$ such that

$$
\left|f(\sigma(t))-f(s)-f^{\Delta}(t)(\sigma(t)-s)\right| \leq \epsilon|\sigma(t)-s| \quad \forall s \in U .
$$


For $f: \mathbb{T} \rightarrow \mathbb{R}$ and $t \in \mathbb{T}_{\kappa}$, then the nabla derivative of $f$ at the point $t$ is defined to be the number $f^{\nabla}(t)$ (provided it exists) with the property that for each $\epsilon>0$, there is a neighborhood $U$ of $t$ such that

$$
\left|f(\rho(t))-f(s)-f^{\nabla}(t)(\rho(t)-s)\right| \leq \epsilon|\rho(t)-s| \quad \forall s \in U
$$

Definition 2.3 (see [8]). A function $f$ is rd-continuous provided it is continuous at each rightdense point in $\mathbb{T}$ and has a left-sided limit at each left-dense point in $\mathbb{T}$. The set of rdcontinuous functions $f$ will be denoted by $C_{\mathrm{rd}}(\mathbb{T})$. A function $g$ is left-dense continuous (i.e., ld-continuous) if $g$ is continuous at each left-dense point in $\mathbb{T}$ and its right-sided limit exists (finite) at each right-dense point in $\mathbb{T}$. The set of left-dense continuous functions $g$ will be denoted by $C_{\mathrm{ld}}(\mathbb{T})$.

Definition 2.4 (see [8]). If $F^{\Delta}(t)=f(t)$, then we define the delta integral by

$$
\int_{a}^{b} f(s) \Delta s=F(b)-F(a)
$$

If $G^{\nabla}(t)=g(t)$, then we define the nabla integral by

$$
\int_{a}^{b} g(s) \nabla s=G(b)-G(a) .
$$

Lemma 2.5 (see $[8]$ ). If $f \in C_{\mathrm{rd}}(\mathbb{T})$ and $t \in \mathbb{T}^{\kappa}$, then

$$
\int_{t}^{\sigma(t)} f(s) \Delta s=\mu(t) f(t)
$$

If $g \in C_{\mathrm{ld}}(\mathbb{T})$ and $t \in \mathbb{T}_{\kappa}$, then

$$
\int_{\rho(t)}^{t} g(s) \nabla s=v(t) g(t)
$$

Let the Banach space

$$
\begin{aligned}
\mathbb{B} & =C_{1 \mathrm{~d}}^{1}\left([0, T]_{\mathbb{T}}\right) \\
& =\left\{x:[0, T]_{\mathbb{T}} \longrightarrow \mathbb{R} \mid x \text { is } \Delta \text {-differentiable on }[0, T]_{\mathbb{T}}, \text { and } x^{\Delta} \text { is ld-continuous on }[0, T]_{\mathbb{T}}\right\}
\end{aligned}
$$

be endowed with the norm $\|x\|=\max \left\{\|x\|_{0},\left\|x^{\Delta}\right\|_{0}\right\}$, where

$$
\|x\|_{0}=\sup _{t \in[0, T]_{\mathbb{T}}}|x(t)|, \quad\left\|x^{\Delta}\right\|_{0}=\sup _{t \in[0, T]_{\mathbb{T}}}\left|x^{\Delta}(t)\right|
$$


and choose a cone $\mathbb{P} \subset \mathbb{B}$ defined by

$$
\mathbb{P}=\left\{\begin{array}{c}
x \in \mathbb{B}: x(t) \geq 0, \quad x^{\Delta}(t) \leq 0, x^{\Delta \nabla}(t) \leq 0 \quad \forall t \in[0, T]_{\mathbb{T}} \\
\alpha x(T)-\beta x(0)=\int_{0}^{T} g(s) x(s) \nabla s
\end{array}\right\} .
$$

Lemma 2.6. If $x \in \mathbb{P}$, then $x(t) \geq \beta /\left(\alpha-g_{0}\right)\|x\|_{0}$ for all $t \in[0, T]_{\mathbb{T}}$.

Proof. If $x \in \mathbb{P}$, then $x^{\Delta} \leq 0$. It follows that

$$
x(T)=\min _{t \in[0, T]_{\mathbb{T}}} x(t), \quad\|x\|_{0}=x(0)=\max _{t \in[0, T]_{\mathbb{T}}} x(t) .
$$

With $\alpha x(T)-\beta x(0)=\int_{0}^{T} g(s) x(s) \nabla s$ and $x^{\Delta} \leq 0$, one obtains

$$
\alpha x(T)=\beta x(0)+\int_{0}^{T} g(s) x(s) \nabla s \geq \beta x(0)+\int_{0}^{T} g(s) \nabla s x(T)=\beta x(0)+g_{0} x(T) .
$$

Therefore,

$$
x(T) \geq \frac{\beta}{\alpha-g_{0}} x(0)=\frac{\beta}{\alpha-g_{0}}\|x\|_{0} .
$$

From (2.11)-(2.13), we can get that

$$
x(t) \geq \min _{t \in[0, T]_{\mathbb{T}}} x(t)=x(T) \geq \frac{\beta}{\alpha-g_{0}} x(0)=\frac{\beta}{\alpha-g_{0}}\|x\|_{0} .
$$

So Lemma 2.6 is proved.

Lemma 2.7. $x \in \mathbb{B}$ is a solution of BVPs (1.1)-(1.2) if and only if $x \in \mathbb{B}$ is a solution of the following integral equation:

$$
\begin{aligned}
x(t)= & \int_{0}^{T} \Theta(\beta+V(s)) \varphi_{q}\left(\int_{0}^{s} \lambda f\left(r, x(r), x^{\Delta}(r)\right) \nabla r\right) \Delta s \\
& +\int_{t}^{T} \varphi_{q}\left(\int_{0}^{s} \lambda f\left(r, x(r), x^{\Delta}(r)\right) \nabla r\right) \Delta s,
\end{aligned}
$$

where

$$
\begin{gathered}
\Theta=\frac{1}{\alpha-\beta-\int_{0}^{T} g(s) \nabla s}=\frac{1}{\alpha-\beta-g_{0}}, \\
V(t)=\int_{0}^{t} g(s) \nabla s \quad \forall t \in[0, T]_{\mathbb{T}} .
\end{gathered}
$$


Proof. First assume $x \in \mathbb{B}$ is a solution of BVPs (1.1)-(1.2); then we have

$$
\varphi_{p}\left(x^{\Delta}(t)\right)=\varphi_{p}\left(x^{\Delta}(0)\right)-\int_{0}^{t} \lambda f\left(s, x(s), x^{\Delta}(s)\right) \nabla s=-\int_{0}^{t} \lambda f\left(s, x(s), x^{\Delta}(s)\right) \nabla s .
$$

That is,

$$
x^{\Delta}(t)=-\varphi_{q}\left(\int_{0}^{t} \lambda f\left(s, x(s), x^{\Delta}(s)\right) \nabla s\right)=-H(t) .
$$

Integrating (2.18) from $t$ to $T$, it follows that

$$
x(t)=x(T)+\int_{t}^{T} H(s) \Delta s
$$

Together with (2.19) and $\alpha x(T)-\beta x(0)=\int_{0}^{T} g(s) x(s) \nabla s$, we obtain

$$
\alpha x(T)-\beta\left(x(T)+\int_{0}^{T} H(s) \Delta s\right)=\int_{0}^{T} g(s)\left(x(T)+\int_{s}^{T} H(r) \Delta r\right) \nabla s
$$

Thus,

$$
\begin{aligned}
\left(\alpha-\beta-\int_{0}^{T} g(s) \nabla s\right) x(T) & =\beta \int_{0}^{T} H(s) \Delta s+\int_{0}^{T} g(s)\left(\int_{s}^{T} H(r) \Delta r\right) \nabla s \\
& =\beta \int_{0}^{T} H(s) \Delta s+\int_{0}^{T}\left(\int_{s}^{T}(V(s)-V(r)) H(r) \Delta r\right) \nabla s \\
& =\beta \int_{0}^{T} H(s) \Delta s-\int_{0}^{T}(V(0)-V(s)) H(s) \Delta s \\
& =\beta \int_{0}^{T} H(s) \Delta s+\int_{0}^{T} V(s) H(s) \Delta s,
\end{aligned}
$$

namely,

$$
x(T)=\beta \Theta \int_{0}^{T} H(s) \Delta s+\Theta \int_{0}^{T} V(s) H(s) \Delta s .
$$


Substituting (2.22) into (2.19), we obtain

$$
\begin{aligned}
x(t)= & \beta \Theta \int_{0}^{T} H(s) \Delta s+\Theta \int_{0}^{T} V(s) H(s) \Delta s+\int_{t}^{T} H(s) \Delta s \\
= & \int_{0}^{T} \Theta(\beta+V(s)) \varphi_{q}\left(\int_{0}^{s} \lambda f\left(r, x(r), x^{\Delta}(r)\right) \nabla r\right) \Delta s \\
& +\int_{t}^{T} \varphi_{q}\left(\int_{0}^{s} \lambda f\left(r, x(r), x^{\Delta}(r)\right) \nabla r\right) \Delta s .
\end{aligned}
$$

The proof of sufficiency is complete.

Conversely, assume $x \in \mathbb{B}$ is a solution of the following integral equation:

$$
\begin{aligned}
x(t)= & \int_{0}^{T} \Theta(\beta+V(s)) \varphi_{q}\left(\int_{0}^{s} \lambda f\left(r, x(r), x^{\Delta}(r)\right) \nabla r\right) \Delta s \\
& +\int_{t}^{T} \varphi_{q}\left(\int_{0}^{s} \lambda f\left(r, x(r), x^{\Delta}(r)\right) \nabla r\right) \Delta s \\
= & \int_{0}^{T} \Theta(\beta+V(s)) H(s) \Delta s+\int_{t}^{T} H(s) \Delta s .
\end{aligned}
$$

It follows that

$$
\begin{gathered}
x^{\Delta}(t)=-\varphi_{q}\left(\int_{0}^{t} \lambda f\left(s, x(s), x^{\Delta}(s)\right) \nabla s\right)=-H(t), \\
\left(\varphi_{p}\left(x^{\Delta}(t)\right)\right)^{\nabla}+\lambda f\left(t, x(t), x^{\Delta}(t)\right)=0 .
\end{gathered}
$$

So $x^{\Delta}(0)=0$. Furthermore, we have

$$
\begin{aligned}
\alpha x(T)-\beta x(0) & =\alpha \int_{0}^{T} \Theta(\beta+V(s)) H(s) \Delta s-\beta \int_{0}^{T} \Theta(\beta+V(s)) H(s) \Delta s-\beta \int_{0}^{T} H(s) \Delta s \\
& =(\alpha-\beta) \int_{0}^{T} \Theta(\beta+V(s)) H(s) \Delta s-\beta \int_{0}^{T} H(s) \Delta s \\
\int_{0}^{T} g(s) x(s) \nabla s & =\int_{0}^{T} g(s)\left(\int_{0}^{T} \Theta(\beta+V(r)) H(r) \Delta r+\int_{s}^{T} H(r) \Delta r\right) \nabla s \\
& =\int_{0}^{T} g(s) \nabla s \int_{0}^{T} \Theta(\beta+V(s)) H(s) \Delta s+\int_{0}^{T} \int_{s}^{T} g(s) H(r) \Delta r \nabla s
\end{aligned}
$$


Boundary Value Problems

$$
\begin{aligned}
= & \int_{0}^{T} g(s) \nabla s \int_{0}^{T} \Theta(\beta+V(s)) H(s) \Delta s \\
& +\int_{0}^{T}\left(\int_{s}^{T}(V(s)-V(r)) H(r) \Delta r\right) \nabla s \\
= & \int_{0}^{T} g(s) \nabla s \int_{0}^{T} \Theta(\beta+V(s)) H(s) \Delta s+\int_{0}^{T} V(s) H(s) \Delta s,
\end{aligned}
$$

which imply that

$$
\begin{aligned}
\alpha x(T)-\beta x(0)-\int_{0}^{T} g(s) x(s) \nabla s= & (\alpha-\beta) \int_{0}^{T} \Theta(\beta+V(s)) H(s) \Delta s \\
& -\beta \int_{0}^{T} H(s) \Delta s-\int_{0}^{T} g(s) \nabla s \int_{0}^{T} \Theta(\beta+V(s)) H(s) \Delta s \\
& -\int_{0}^{T} V(s) H(s) \Delta s \\
= & 0 .
\end{aligned}
$$

The proof of Lemma 2.7 is complete.

Define the operator $\Psi: \mathbb{P} \rightarrow \mathbb{B}$ by

$$
\begin{aligned}
(\Psi x)(t)= & \int_{0}^{T} \Theta(\beta+V(s)) \varphi_{q}\left(\int_{0}^{s} \lambda f\left(r, x(r), x^{\Delta}(r)\right) \nabla r\right) \Delta s \\
& +\int_{t}^{T} \varphi_{q}\left(\int_{0}^{s} \lambda f\left(r, x(r), x^{\Delta}(r)\right) \nabla r\right) \Delta s
\end{aligned}
$$

for all $t \in[0, T]_{\mathbb{T}}$. Obviously, $\Psi x(t) \geq 0$ for all $t \in[0, T]_{\mathbb{T}}$.

Lemma 2.8. If $x \in \mathbb{P}$, then $\Psi x \in \mathbb{P}$.

Proof. It is easily obtained from the second part of the proof in Lemma 2.7. The proof is complete.

Lemma 2.9. $\Psi: \mathbb{P} \rightarrow \mathbb{P}$ is complete continuous.

Proof. First, we show that $\Psi$ maps bounded set into itself. Assume $c$ is a positive constant and $x \in \overline{\mathbb{P}}_{c}=\{x \in \mathbb{P}:\|x\| \leq c\}$. Note that the continuity of $f\left(t, x, x^{\Delta}\right)$ guarantees that there is a 
$C>0$ such that $f\left(t, x, x^{\Delta}\right) \leq \varphi_{p}(C)$ for all $t \in[0, T]_{\mathbb{T}}$. So we get from $\Psi^{\Delta} x \leq 0$ and $\Psi^{\Delta \nabla} x \leq 0$ that

$$
\begin{aligned}
\|\Psi x\|_{0}= & \Psi x(0) \\
= & \int_{0}^{T} \Theta(\beta+V(s)) \varphi_{q}\left(\int_{0}^{s} \lambda f\left(r, x(r), x^{\Delta}(r)\right) \nabla r\right) \Delta s \\
& +\int_{0}^{T} \varphi_{q}\left(\int_{0}^{s} \lambda f\left(r, x(r), x^{\Delta}(r)\right) \nabla r\right) \Delta s \\
\leq & C \lambda^{q-1} T^{q-1} \int_{0}^{T} \Theta(\beta+V(s)) \Delta s+C \lambda^{q-1} T^{q}, \\
\left\|\Psi^{\Delta} x\right\|_{0}= & \left|\Psi^{\Delta} x(T)\right| \\
= & \varphi_{q}\left(\int_{0}^{T} \lambda f\left(r, x(r), x^{\Delta}(r)\right) \nabla r\right) \\
\leq & C \lambda^{q-1} T^{q-1} .
\end{aligned}
$$

That is, $\Psi \overline{\mathbb{P}}_{c}$ is uniformly bounded. In addition, notice that

$$
\begin{aligned}
\left|(\Psi x)\left(t_{1}\right)-(\Psi x)\left(t_{2}\right)\right| & =\left|\int_{t_{2}}^{t_{1}} \varphi_{q}\left(\int_{0}^{s} \lambda f\left(r, x(r), x^{\Delta}(r)\right) \nabla r\right) \Delta s\right| \\
& \leq C \lambda^{q-1} T^{q-1}\left|t_{1}-t_{2}\right|
\end{aligned}
$$

which implies that

$$
\begin{aligned}
\left|(\Psi x)\left(t_{1}\right)-(\Psi x)\left(t_{2}\right)\right| \longrightarrow 0 & \text { as } t_{1}-t_{2} \longrightarrow 0, \\
\left|\left((\Psi x)^{\Delta}\left(t_{1}\right)\right)^{p-1}-\left((\Psi x)^{\Delta}\left(t_{2}\right)\right)^{p-1}\right| & =\left|\varphi_{p}\left((\Psi x)^{\Delta}\left(t_{1}\right)\right)-\varphi_{p}\left((\Psi x)^{\Delta}\left(t_{2}\right)\right)\right| \\
& =\left|\int_{t_{2}}^{t_{1}} \lambda f\left(r, x(r), x^{\Delta}(r)\right) \nabla r\right| \\
& \leq \lambda \varphi_{p}(C)\left|t_{1}-t_{2}\right|,
\end{aligned}
$$

which implies that

$$
\left|\left((\Psi x)^{\Delta}\left(t_{1}\right)\right)^{p-1}-\left((\Psi x)^{\Delta}\left(t_{2}\right)\right)^{p-1}\right| \longrightarrow 0 \quad \text { as } t_{1}-t_{2} \longrightarrow 0
$$

That is,

$$
\left|(\Psi x)^{\Delta}\left(t_{1}\right)-(\Psi x)^{\Delta}\left(t_{2}\right)\right| \longrightarrow 0 \quad \text { as } t_{1}-t_{2} \longrightarrow 0
$$


So $\Psi x$ is equicontinuous for any $x \in \overline{\mathbb{P}}_{c}$. Using Arzela-Ascoli theorem on time scales [17], we obtain that $\Psi \overline{\mathbb{P}}_{c}$ is relatively compact. In view of Lebesgue's dominated convergence theorem on time scales [18], it is easy to prove that $\Psi$ is continuous. Hence, $\Psi$ is complete continuous. The proof of this lemma is complete.

Let $v$ and $\omega$ be nonnegative continuous convex functionals on a pone $\mathbb{P}, \psi$ a nonnegative continuous concave functional on $\mathbb{P}$, and $r, a, L$ positive numbers with $r>a$ we defined the following convex sets:

$$
\begin{gathered}
\mathbb{P}(v, r ; \omega, l)=\{x \in \mathbb{P}: v(x)<r, \omega(x)<l\}, \\
\overline{\mathbb{P}}(v, r ; \omega, l)=\{x \in \mathbb{P}: v(x) \leq r, \omega(x) \leq l\}, \\
\mathbb{P}(v, r ; \omega, l ; \psi, a)=\{x \in \mathbb{P}: v(x)<r, \omega(x)<l, \psi(x)>a\}, \\
\overline{\mathbb{P}}(v, r ; \omega, l ; \psi, a)=\{x \in \mathbb{P}: v(x) \leq r, \omega(x) \leq l, \psi(x) \geq a\}
\end{gathered}
$$

and introduce two assumptions with regard to the functionals $v, w$ as follows:

(H1) there exists $M>0$ such that $\|x\| \leq M \max \{v(x), \omega(x)\}$ for all $x \in \mathbb{P}$;

(H2) $\mathbb{P}(v, r ; \omega, l) \neq \emptyset$ for any $r>0$ and $l>0$.

The following fixed point theorem duo to Bai and Ge is crucial in the arguments of our main result.

Lemma 2.10 (see [19]). Let $\mathbb{B}$ be Banach space, $\mathbb{P} \subset \mathbb{B}$ a cone, and $r_{2} \geq d>b>r_{1}>0, l_{2} \geq l_{1}>0$. Assume that $v$ and $\omega$ are nonnegative continuous convex functionals satisfying (H1) and (H2), $\psi$ is a nonnegative continuous concave functional on $\mathbb{P}$ such that $\psi(x) \leq v(x)$ for all $x \in \overline{\mathbb{P}}\left(v, r_{2} ; \omega, l_{2}\right)$, and $\Psi: \overline{\mathbb{P}}\left(v, r_{2} ; \omega, l_{2}\right) \rightarrow \overline{\mathbb{P}}\left(v, r_{2} ; \omega, l_{2}\right)$ is a complete continuous operator. Suppose

(C1) $\left\{x \in \overline{\mathbb{P}}\left(v, d ; \omega, l_{2} ; \psi, b\right)\right\} \neq \emptyset, \psi(\Psi x)>b$ for $x \in \overline{\mathbb{P}}\left(v, d ; \omega, l_{2} ; \psi, b\right)$;

(C2) $v(\Psi x)<r_{1}, \omega(\Psi x)<l_{1}$ for $x \in \overline{\mathbb{P}}\left(v, r_{1} ; \omega, l_{1}\right)$;

(C3) $\psi(\Psi x)>b$ for $x \in \overline{\mathbb{P}}\left(v, r_{2} ; \omega, l_{2} ; \psi, b\right)$ with $v(\Psi x)>d$.

Then $\Psi$ has at least three fixed points $x_{1}, x_{2}, x_{3} \in \overline{\mathbb{P}}\left(v, r_{2} ; \omega, l_{2}\right)$ with

$$
\begin{gathered}
x_{1} \in \mathbb{P}\left(v, r_{1} ; \omega, l_{1}\right), \\
x_{2} \in\left\{x \in \overline{\mathbb{P}}\left(v, r_{2} ; \omega, l_{2} ; \psi, b\right): \psi(x)>b\right\}, \\
x_{3} \in \overline{\mathbb{P}}\left(v, r_{2} ; \omega, l_{2}\right) \backslash\left(\overline{\mathbb{P}}\left(v, r_{2} ; \omega, l_{2} ; \psi, b\right) \cup \overline{\mathbb{P}}\left(v, r_{1} ; \omega, l_{1}\right)\right) .
\end{gathered}
$$

\section{Main Result}

In this section, we will give sufficient conditions for the existence of at least three positive solutions to BVPs (1.1)-(1.2). 
Theorem 3.1. Suppose that there are positive numbers $0<\epsilon_{0}<\epsilon<T, l_{2} \geq l_{1}>0$, and $r_{2}>b>$ $r_{1}>0$ with $\epsilon_{0}, \epsilon \in[0, T]_{\mathbb{T}}, b / N \leq \min \left\{r_{2} / K, l_{2} / L\right\}$ and $\alpha b-g_{0} b \leq r_{2} \beta$ such that the following conditions are satisfied.

(H3) $f(t, u, v) \leq \min \left\{\varphi_{p}\left(r_{2} / K\right), \varphi_{p}\left(l_{2} / L\right)\right\}$ for all $(t, u, v) \in[0, T]_{\mathbb{T}} \times\left[0, r_{2}\right] \times\left[-l_{2}, l_{2}\right]$, where

$$
K=\lambda^{q-1}\left(\int_{0}^{T} \Theta(\beta+V(s)) s^{q-1} \Delta s+\int_{0}^{T} s^{q-1} \Delta s\right), \quad L=\lambda^{q-1} T^{q-1} .
$$

(H4) $f(t, u, v)<\min \left\{\varphi_{p}\left(r_{1} / K\right), \varphi_{p}\left(l_{1} / L\right)\right\}$ for all $(t, u, v) \in[0, T]_{\mathbb{T}} \times\left[0, r_{1}\right] \times\left[-l_{1}, l_{1}\right]$.

(H5) $f(t, u, v)>\varphi_{p}(b / N)$ for all $(t, u, v) \in\left[\epsilon_{0}, \epsilon\right]_{\mathbb{T}} \times\left[b,\left(\alpha b-g_{0} b\right) / \beta\right] \times\left[-l_{2}, l_{2}\right]$, where

$$
N=\lambda^{q-1}\left(\epsilon-\epsilon_{0}\right)^{q-1} \int_{\epsilon}^{T} \Theta(\beta+V(s)) \Delta s
$$

Then BVPs (1.1)-(1.2) have at least three positive solutions.

Proof. By the definition of the operator $\Psi$ and its properties, it suffices to show that the conditions of Lemma 2.10 hold with respect to the operator $\Psi$.

Let the nonnegative continuous convex functionals $v, w$ and the nonnegative continuous concave functional $\psi$ be defined on the cone $\mathbb{P}$ by

$$
v(x)=\max _{t \in[0, T]_{\mathbb{T}}}|x(t)|=x(0), \quad \omega(x)=\max _{t \in[0, T]_{\mathbb{T}}}\left|x^{\Delta}(t)\right|=x^{\Delta}(T), \quad \psi(x)=\min _{t \in[\epsilon, T]_{\mathbb{T}}} x(t)=x(T) .
$$

Then it is easy to see that $\|x\|=\max \{v(x), \omega(x)\}$ and (H1)-(H2) hold. then

First of all, we show that $\Psi: \overline{\mathbb{P}}\left(v, r_{2} ; \omega, l_{2}\right) \rightarrow \overline{\mathbb{P}}\left(v, r_{2} ; \omega, l_{2}\right)$. In fact, if $x \in \overline{\mathbb{P}}\left(v, r_{2} ; \omega, l_{2}\right)$,

$$
v(x)=\max _{t \in[0, T]_{\mathbb{T}}}|x(t)| \leq r_{2}, \quad \omega(x)=\max _{t \in[0, T]_{\mathbb{T}}}\left|x^{\Delta}(t)\right| \leq l_{2}
$$

and assumption (H3) implies that

$$
f\left(t, x(t), x^{\Delta}(t)\right) \leq \min \left\{\varphi_{p}\left(\frac{r_{2}}{K}\right), \varphi_{p}\left(\frac{l_{2}}{L}\right)\right\} \quad \forall t \in[0, T]_{\mathbb{T}} .
$$


On the other hand, for $x \in \mathbb{P}$, there is $\Psi x \in \mathbb{P}$; thus

$$
\begin{aligned}
& v(\Psi x)=\max _{t \in[0, T]_{\mathbb{T}}}|(\Psi x)(t)| \\
& =\max _{t \in[0, T]_{\mathbb{T}}} \mid \int_{0}^{T} \Theta(\beta+V(s)) \varphi_{q}\left(\int_{0}^{s} \lambda f\left(r, x(r), x^{\Delta}(r)\right) \nabla r\right) \Delta s \\
& +\int_{t}^{T} \varphi_{q}\left(\int_{0}^{s} \lambda f\left(r, x(r), x^{\Delta}(r)\right) \nabla r\right) \Delta s \\
& =\mid \int_{0}^{T} \Theta(\beta+V(s)) \varphi_{q}\left(\int_{0}^{s} \lambda f\left(r, x(r), x^{\Delta}(r)\right) \nabla r\right) \Delta s \\
& +\int_{0}^{T} \varphi_{q}\left(\int_{0}^{s} \lambda f\left(r, x(r), x^{\Delta}(r)\right) \nabla r\right) \Delta s \\
& \leq \int_{0}^{T} \Theta(\beta+V(s)) \varphi_{q}\left(\int_{0}^{s} \lambda \varphi_{p}\left(\frac{r_{2}}{K}\right) \nabla r\right) \Delta s \\
& +\int_{0}^{T} \varphi_{q}\left(\int_{0}^{s} \lambda \varphi_{p}\left(\frac{r_{2}}{K}\right) \nabla r\right) \Delta s \\
& =\frac{r_{2}}{K} \int_{0}^{T} \Theta(\beta+V(s)) \varphi_{q}\left(\int_{0}^{s} \lambda \nabla r\right) \Delta s+\frac{r_{2}}{K} \int_{0}^{T} \varphi_{q}\left(\int_{0}^{s} \lambda \nabla r\right) \Delta s \\
& =\frac{r_{2}}{K} \lambda^{q-1}\left(\int_{0}^{T} \Theta(\beta+V(s)) s^{q-1} \Delta s+\int_{0}^{T} s^{q-1} \Delta s\right) \\
& =\frac{r_{2}}{K} \cdot K \\
& =r_{2} \text {, } \\
& \omega(\Psi x)=\max _{t \in[0, T]_{\mathbb{T}}}\left|(\Psi x)^{\Delta}(t)\right| \\
& =\max _{t \in[0, T]_{\mathbb{T}}}\left|-\varphi_{q}\left(\int_{0}^{t} \lambda f\left(r, x(r), x^{\Delta}(r)\right) \nabla r\right)\right| \\
& =\varphi_{q}\left(\int_{0}^{T} \lambda f\left(r, x(r), x^{\Delta}(r)\right) \nabla r\right) \\
& \leq \varphi_{q}\left(\int_{0}^{T} \lambda \varphi_{p}\left(\frac{l_{2}}{L}\right) \nabla r\right) \\
& =\frac{l_{2}}{L} \varphi_{q}\left(\int_{0}^{T} \lambda \nabla r\right) \\
& =l_{2} \text {. }
\end{aligned}
$$

Therefore, $\Psi: \overline{\mathbb{P}}\left(v, r_{2} ; \omega, l_{2}\right) \rightarrow \overline{\mathbb{P}}\left(v, r_{2} ; \omega, l_{2}\right)$. 
In the same way, if $x \in \overline{\mathbb{P}}\left(v, r_{1} ; \omega, l_{1}\right)$, then assumption (H4) implies

$$
f\left(t, x(t), x^{\Delta}(t)\right)<\min \left\{\varphi_{p}\left(\frac{r_{1}}{K}\right), \varphi_{p}\left(\frac{l_{1}}{L}\right)\right\} \quad \forall t \in[0, \mathrm{~T}]_{\mathbb{T}} .
$$

As in the argument above, we can get that $\Psi: \overline{\mathbb{P}}\left(v, r_{1} ; \omega, l_{1}\right) \rightarrow \overline{\mathbb{P}}\left(v, r_{1} ; \omega, l_{1}\right)$. Thus, condition (C2) of Lemma 2.10 holds.

To check condition (C1) in Lemma 2.10. Let $d=\left(\alpha b-g_{0} b\right) / \beta$. We choose $x(t) \equiv d>b$ for $t \in[0, T]_{\mathbb{T}}$. It is easy to see that

$$
x(t) \equiv d \in \overline{\mathbb{P}}\left(v, d ; \omega, l_{2} ; \psi, b\right), \quad \psi(x)=d>b .
$$

Consequently,

$$
\left\{x \in \overline{\mathbb{P}}\left(v, d ; \omega, l_{2} ; \psi, b\right): \psi(x)>b\right\} \neq \emptyset .
$$

Hence, for $x \in \overline{\mathbb{P}}\left(v, d ; \omega, l_{2} ; \psi, b\right)$, there are

$$
b \leq x(t) \leq d, \quad\left|x^{\Delta}(t)\right| \leq l_{2} \quad \forall t \in[\epsilon, T]_{\mathbb{T}} .
$$

In view of assumption (H5), we have

$$
f\left(t, x(t), x^{\Delta}(t)\right)>\varphi_{p}\left(\frac{b}{N}\right) \quad \forall t \in\left[\epsilon_{0}, \epsilon\right]_{\mathbb{T}} .
$$

It follows that

$$
\begin{aligned}
\psi(\Psi x) & =\min _{t \in[\epsilon, T]_{\mathbb{T}}}(\Psi x)(t) \\
& =(\Psi x)(T) \\
& =\int_{0}^{T} \Theta(\beta+V(s)) \varphi_{q}\left(\int_{0}^{s} \lambda f\left(r, x(r), x^{\Delta}(r)\right) \nabla r\right) \Delta s \\
& \geq \int_{\epsilon}^{T} \Theta(\beta+V(s)) \varphi_{q}\left(\int_{0}^{s} \lambda f\left(r, x(r), x^{\Delta}(r)\right) \nabla r\right) \Delta s \\
& \geq \int_{\epsilon}^{T} \Theta(\beta+V(s)) \varphi_{q}\left(\int_{0}^{\epsilon} \lambda f\left(r, x(r), x^{\Delta}(r)\right) \nabla r\right) \Delta s \\
& \geq \int_{\epsilon}^{T} \Theta(\beta+V(s)) \varphi_{q}\left(\int_{\epsilon_{0}}^{\epsilon} \lambda f\left(r, x(r), x^{\Delta}(r)\right) \nabla r\right) \Delta s \\
& >\int_{\epsilon}^{T} \Theta(\beta+V(s)) \varphi_{q}\left(\int_{\epsilon_{0}}^{\epsilon} \lambda \varphi_{p}\left(\frac{b}{N}\right) \nabla r\right) \Delta s
\end{aligned}
$$




$$
\begin{aligned}
& =\lambda^{q-1}\left(\epsilon-\epsilon_{0}\right)^{q-1} \int_{\epsilon}^{T} \Theta(\beta+V(s)) \Delta s \frac{b}{N} \\
& =N \cdot \frac{b}{N} \\
& =b .
\end{aligned}
$$

Therefore, $\psi(\Psi x)>b$ for $x \in \overline{\mathbb{P}}\left(v, d ; \omega, l_{2} ; \psi, b\right)$. So condition (C1) in Lemma 2.10 is satisfied.

Finally, we show that (C3) in Lemma 2.10 holds. In fact, for $x \in \overline{\mathbb{P}}\left(v, r_{2} ; \omega, l_{2} ; \psi, b\right)$ and $v(\Psi x)>d=\left(\alpha b-g_{0} b\right) / \beta$, we have

$$
\psi(\Psi x)=\min _{t \in[\epsilon, T]_{\mathbb{T}}}(\Psi x)(t)=(\Psi x)(T) \geq \frac{\beta}{\alpha-g_{0}} \max _{t \in[0, T]_{\mathbb{T}}}(\Psi x)(t)=\frac{\beta}{\alpha-g_{0}} v(\Psi x)>b
$$

Thus by Lemma 2.10 and the assumption that $f(t, 0,0) \neq 0$ on $[0, T]_{\mathbb{T}}$, BVPs (1.1)-(1.2) have at least three positive solutions. The proof is complete.

Theorem 3.2. Suppose that there are positive numbers $0<\xi<T, l_{2} \geq l_{1}>0$, and $r_{2}>b>r_{1}>0$ with $\xi \in[0, T]_{\mathbb{T}}, b / F \leq \min \left\{r_{2} / K, l_{2} / L\right\}$, and $\alpha b-g_{0} b \leq r_{2} \beta$ such that (H3)-(H4) and the following condition are satisfied.

(H6) $f(t, u, v)>\varphi_{p}(b / F)$ for all $(t, u, v) \in[0, \xi]_{\mathbb{T}} \times\left[b,\left(\alpha b-g_{0} b\right) / \beta\right] \times\left[-l_{2}, l_{2}\right]$, where

$$
F=\lambda^{q-1} \xi^{q-1}(T-\xi)
$$

Then BVPS (1.1)-(1.2) have at least three positive solutions.

Proof. Let the nonnegative continuous convex functionals $v, \omega$ be defined on the cone $\mathbb{P}$ as Theorem 3.1 and the nonnegative continuous concave functional $\psi$ be defined on the cone $\mathbb{P}$ by

$$
\psi(x)=\min _{t \in[0, \xi]_{\mathbb{T}}} x(t)=x(\xi) .
$$

We will show that condition (C1) in Lemma 2.10 holds. Let $d=\left(\alpha b-g_{0} b\right) / \beta$. We choose $x(t) \equiv d>b$ for $t \in[0, T]_{\mathbb{T}}$. It is easy to see that

$$
x(t) \equiv d \in \overline{\mathbb{P}}\left(v, d ; \omega, l_{2} ; \psi, b\right), \quad \psi(x)=d>b .
$$

Consequently,

$$
\left\{x \in \overline{\mathbb{P}}\left(v, d ; \omega, l_{2} ; \psi, b\right): \psi(x)>b\right\} \neq \emptyset .
$$


Hence, for $x \in \overline{\mathbb{P}}\left(v, d ; \omega, l_{2} ; \psi, b\right)$, there are

$$
b \leq x(t) \leq d, \quad\left|x^{\Delta}(t)\right| \leq l_{2} \quad \forall t \in[0, \xi]_{\mathbb{T}} .
$$

In view of assumption (H6), we have

$$
f\left(t, x(t), x^{\Delta}(t)\right)>\varphi_{p}\left(\frac{b}{F}\right) \quad \forall t \in[0, \xi]_{\mathbb{T}} .
$$

It follows that

$$
\begin{aligned}
\psi(\Psi x)= & \min _{t \in[0, \xi]_{\mathbb{T}}}(\Psi x)(t) \\
= & (\Psi x)(\xi) \\
= & \int_{0}^{T} \Theta(\beta+V(s)) \varphi_{q}\left(\int_{0}^{s} \lambda f\left(r, x(r), x^{\Delta}(r)\right) \nabla r\right) \Delta s \\
& +\int_{\xi}^{T} \varphi_{q}\left(\int_{0}^{s} \lambda f\left(r, x(r), x^{\Delta}(r)\right) \nabla r\right) \Delta s \\
\geq & \int_{\xi}^{T} \varphi_{q}\left(\int_{0}^{s} \lambda f\left(r, x(r), x^{\Delta}(r)\right) \nabla r\right) \Delta s \\
\geq & \int_{\xi}^{T} \varphi_{q}\left(\int_{0}^{\xi} \lambda f\left(r, x(r), x^{\Delta}(r)\right) \nabla r\right) \Delta s \\
> & \int_{\xi}^{T} \varphi_{q}\left(\int_{0}^{\xi} \lambda \varphi_{p}\left(\frac{b}{F}\right) \nabla r\right) \Delta s \\
= & \lambda^{q-1} \xi^{q-1}(T-\xi) \frac{b}{F} \\
= & F \cdot \frac{b}{F} \\
= & b .
\end{aligned}
$$

Therefore, $\psi(\Psi x)>b$ for $x \in \overline{\mathbb{P}}\left(v, d ; \omega, l_{2} ; \psi, b\right)$. So condition (C1) in Lemma 2.10 is satisfied. Using a similar proof to Theorem 3.1, the other conditions in Lemma 2.10 are satisfied. By Lemma 2.10, BVPs (1.1)-(1.2) have at least three positive solutions. The proof is complete.

\section{An Example}

Example 4.1. Consider the following second-order Laplacian dynamic equations on time scales

$$
\left(\varphi_{1.5}\left(x^{\Delta}(t)\right)\right)^{\nabla}+f\left(t, x(t), x^{\Delta}(t)\right)=0, \quad t \in(0,1)_{\mathbb{T}}
$$


with integral boundary condition

$$
x^{\Delta}(0)=0, \quad 3 x(1)-x(0)=\int_{0}^{1} e^{s-1} x(s) \nabla s,
$$

where

$$
f(t, u, v)= \begin{cases}10^{-5}(t+5|v|)+6|u| & \forall(t, u, v) \in\left([0,1]_{\mathbb{T}} \times[0,12] \times(-\infty,+\infty)\right), \\ 10^{-5}(t+5|v|)+72 & \forall(t, u, v) \in\left([0,1]_{\mathbb{T}} \times(12,+\infty] \times(-\infty,+\infty)\right) .\end{cases}
$$

Then BVPs (4.1)-(4.2) have at least three positive solutions.

Proof. Take $\epsilon_{0}=0.25, \epsilon=0.5, r_{1}=l_{1}=0.009, r_{2}=30000, l_{2}=10000$, and $b=4$. It follows that

$$
\begin{gathered}
\Theta=\frac{1}{\alpha-\beta-g_{0}}=\frac{1}{3-1-\int_{0}^{1} e^{s-1} \nabla s} \leq \frac{1}{3-1-1}=1, \\
\Theta=\frac{1}{\alpha-\beta-g_{0}}=\frac{1}{3-1-\int_{0}^{1} e^{s-1} \nabla s} \geq \frac{1}{3-1-e^{-1}}=0.5 .
\end{gathered}
$$

From (4.1)-(4.2), it is easy to obtain

$$
\begin{gathered}
V(t)=\int_{0}^{t} g(s) \nabla s=\int_{0}^{t} e^{s-1} \nabla s \leq 1 \quad \forall t \in[0,1]_{\mathbb{T}}, \\
V(t)=\int_{0}^{t} g(s) \nabla s=\int_{0}^{t} e^{s-1} \nabla s \geq e^{-1} \geq 0.25 \quad \forall t \in[0,1]_{\mathbb{T}}, \\
K=\int_{0}^{1} \Theta(1+V(s)) s^{3-1} \Delta s+\int_{0}^{1} s^{3-1} \Delta s \leq 3=\bar{K}, \quad L=1, \\
N=(0.5-0.25)^{3-1} \int_{0.5}^{1} \Theta(1+V(s)) \Delta s \leq 0.07=\bar{N} \\
N=(0.5-0.25)^{3-1} \int_{0.5}^{1} \Theta(1+V(s)) \Delta s>0.01 .
\end{gathered}
$$

Hence, we have

$$
\begin{gathered}
\frac{b}{N} \leq 400<10000=\min \left\{\frac{r_{2}}{K}, \frac{l_{2}}{L}\right\}, \\
\alpha b-g_{0} b-r_{2} \beta \leq 12-30000<0 .
\end{gathered}
$$

Moreover, we have 
(H3) for all $(t, u, v) \in[0,1]_{\mathbb{T}} \times[0,30000] \times[-10000,10000]$,

$$
f(t, u, v)<80<100=\min \left\{\varphi_{1.5}\left(\frac{r_{2}}{\bar{K}}\right), \varphi_{1.5}\left(\frac{l_{2}}{L}\right)\right\} \leq \min \left\{\varphi_{p}\left(\frac{r_{2}}{K}\right), \varphi_{p}\left(\frac{l_{2}}{L}\right)\right\}
$$

(H4) for all $(t, u, v) \in[0,1]_{\mathbb{T}} \times[0,0.009] \times[-0.009,0.009]$,

$f(t, u, v) \leq 0.05401045<\min \left\{\varphi_{1.5}\left(\frac{r_{1}}{\bar{K}}\right), \varphi_{1.5}\left(\frac{l_{1}}{L}\right)\right\} \leq \min \left\{\varphi_{p}\left(\frac{r_{1}}{K}\right), \varphi_{p}\left(\frac{l_{1}}{L}\right)\right\} ;$

(H5) for all $(t, u, v) \in[0.25,0.5]_{\mathbb{T}} \times[4,12] \times[-10000,10000]$,

$$
f(t, u, v) \geq 6|u| \geq 24>\varphi_{1.5}\left(\frac{b}{N}\right) .
$$

Therefore, conditions (H3)-(H5) in Theorem 3.1 are satisfied. Further, it is easy to verify that the other conditions in Theorem 3.1 hold. By Theorem 3.1, BVPs (4.1)-(4.2) have at least three positive solutions. The proof is complete.

\section{Acknowledgment}

This work is supported the by the National Natural Sciences Foundation of China under Grant no. 10971183.

\section{References}

[1] D. O'Regan, "Some general existence principles and results for $\left(\varphi\left(y^{\prime}\right)\right)^{\prime}=q f\left(t, y, y^{\prime}\right), 0<t<1$, " SIAM Journal on Mathematical Analysis, vol. 24, no. 3, pp. 648-668, 1993.

[2] M. del Pino, P. Drábek, and R. Manásevich, "The Fredholm alternative at the first eigenvalue for the one-dimensional p-Laplacian," Journal of Differential Equations, vol. 151, no. 2, pp. 386-419, 1999.

[3] A. Cabada and R. L. Pouso, "Existence results for the problem $\left(\varphi\left(y^{\prime}\right)\right)^{\prime}=f\left(t, y, y^{\prime}\right)$ with nonlinear boundary conditions," Nonlinear Analysis: Theory, Methods \& Applications, vol. 35, no. 2, pp. 221-231, 1999.

[4] H. Lü and C. Zhong, "A note on singular nonlinear boundary value problems for the one-dimensional p-Laplacian," Applied Mathematics Letters, vol. 14, no. 2, pp. 189-194, 2001.

[5] W. Feng and J. R. L. Webb, "Solvability of three point boundary value problems at resonance," Nonlinear Analysis: Theory, Methods E Applications, vol. 30, no. 6, pp. 3227-3238, 1997.

[6] C. P. Gupta, "A non-resonant multi-point boundary-value problem for a $p$-Laplacian type operator," in Proceedings of the 5th Mississippi State Conference on Differential Equations and Computational Simulations (Mississippi State, MS, 2001), vol. 10 of Electron. J. Differ. Equ. Conf., pp. 143-152, Southwest Texas State University.

[7] Y. Tian and W. Ge, "Periodic solutions of non-autonomous second-order systems with a $p$-Laplacian," Nonlinear Analysis: Theory, Methods \& Applications, vol. 66, no. 1, pp. 192-203, 2007.

[8] M. Bohner and A. Peterson, Dynamic Equations on Time Scales: An Introduction with Applications, Birkhäuser, Boston, Mass, USA, 2001.

[9] M. Bohner and A. Peterson, Eds., Advances in Dynamic Equations on Time Scales, Birkhäuser, Boston, Mass, USA, 2003. 
[10] R. P. Agarwal, M. Bohner, and W.-T. Li, Nonoscillation and Oscillation: Theory for Functional Differential Equations, vol. 267 of Monographs and Textbooks in Pure and Applied Mathematics, Marcel Dekker, New York, NY, USA, 2004.

[11] H.-R. Sun, L.-T. Tang, and Y.-H. Wang, "Eigenvalue problem for $p$-Laplacian three-point boundary value problems on time scales," Journal of Mathematical Analysis and Applications, vol. 331, no. 1, pp. 248-262, 2007.

[12] L. Jiang and Z. Zhou, "Existence of weak solutions of two-point boundary value problems for secondorder dynamic equations on time scales," Nonlinear Analysis: Theory, Methods E Applications, vol. 69, no. 4, pp. 1376-1388, 2008.

[13] Y. Tian and W. Ge, "Existence and uniqueness results for nonlinear first-order three-point boundary value problems on time scales," Nonlinear Analysis: Theory, Methods E Applications, vol. 69, no. 9, pp. 2833-2842, 2008.

[14] N. Aykut Hamal and Fulya Yoruk, "Positive solutions of nonlinear m-point boundary value problems on time scales," Journal of Computational and Applied Mathematics, vol. 231, no. 1, pp. 92-105, 2009.

[15] H.-R. Sun, "Triple positive solutions for $p$-Laplacian $m$-point boundary value problem on time scales," Computers \& Mathematics with Applications, vol. 58, no. 9, pp. 1736-1741, 2009.

[16] Y. Yang and F. Meng, "Positive solutions of the singular semipositone boundary value problem on time scales," Mathematical and Computer Modelling, vol. 52, no. 3-4, pp. 481-489, 2010.

[17] R. P. Agarwal, M. Bohner, and P. Rehák, "Half-linear dynamic equations," in Nonlinear Analysis and Applications: To V. Lakshmikantham on His 80th Birthday, vol. 1, pp. 1-57, Kluwer Academic Publishers, Dordrecht, The Netherlands, 2003.

[18] B. Aulbach and L. Neidhart, "Integration on measure chains," in Proceedings of the 6th International Conference on Difference Equations, pp. 239-252, CRC Press, Boca Raton, Fla, USA.

[19] Z. Bai and W. Ge, "Existence of three positive solutions for some second-order boundary value problems," Computers \& Mathematics with Applications, vol. 48, no. 5-6, pp. 699-707, 2004. 